\title{
Probabilistic Eye Contact Detection for the Robot-assisted ASD Diagnostic Protocol
}

\author{
Frano Petric, Damjan Miklić and Zdenko Kovačić \\ University of Zagreb, Faculty of Electrical Engineering and Computing \\ Unska 3, 10000 Zagreb, Croatia
}

\begin{abstract}
This paper describes a probabilistic method for eye contact detection, aimed at facilitating the diagnostic process of Autism Spectrum Disorder (ASD). Eye contact, which is a special case of a wider social-attention cue called gaze, plays a major role in ASD diagnostics protocols used in clinical practice. Therefore, a reliable method for automatic eye contact detection is a key capability to open the way towards robotassisted autism diagnostics, which has the potential to reduce diagnostic time and increase reliability. The proposed method uses data from a simple low-resolution monocular camera, which is built into the NAO humanoid robot, and uses head pose and gaze direction as its inputs, which are very prone to outliers. We use a probabilistic framework in order to provide continuous measure of the eye contact and increase robustness to outliers. Furthermore, we take into account the temporal aspect of eye contact, in order to discard short glances which do not indicate actual transfer of attention. Initial experimental results, conducted in a laboratory setting, confirm that the proposed method can be effective in detecting eye contact with the NAO humanoid robot.
\end{abstract}

\section{INTRODUCTION}

Autism Spectrum Disorder (ASD) is a neurodevelopmental disorder characterised by impairments in social interaction, verbal and non-verbal communication, accompanied by repetitive behaviours and interests. With increasing prevalence rates, it is expected to become one of the most commonly diagnosed disorders. To increase the inclusion rate and reduce the cost of lifelong care for people with ASD, experts focus on early diagnostics and intervention, which is hindered by the lack of medical markers that could be used in a diagnostic process. The diagnosis relies on behavioural observations made by experienced clinicians, obtained through several approaches: a) using criteria from the Diagnostic and Statistical Manual of Mental Disorders (DSM) [1]; b) testing children using the Autism Diagnostic Observation Schedule (ADOS) [2]; and, c) interviews with the caregivers using the Autism Diagnostic Interview-Revised (ADI-R) [3].

The diagnostic procedure is highly complex due to simultaneous observation, coding and interpretation of many behaviours as well as administration of various specific tasks, lowering the reliability of the diagnostics and prolonging the time needed to obtain the diagnosis [4]. While ASD can be somewhat reliably identified in children by the age of two, authors in [5] state that there is a need for more continuous measures of aspects of ASD. Modern robotics technologies, similar to those that are already in use in the intervention process [6][7], seem capable of providing adequate tools to address the need for a more objective approach that would help clinicians in gathering multi-modal information and coding the social behaviour, as well as provide the consistent stimuli for interaction.

One of the most important aspects of ASD, heavily relied upon in diagnostics, is the eye contact (or absence of it), as can be inferred from the ADOS protocol [2], in which the eye contact is constantly tracked and quantified. Eye contact is a special case of a wider social attention cue called gaze, where the gaze is directed towards another person's eyes, and can be detected by children as early as 4 months after birth [8].

Detection of eye contact plays a major role in the robotassisted ASD diagnostic protocol [9], [10] which is based on ADOS and aimed at expediting the diagnostics procedure while retaining (and possibly increasing) the reliability of the standard ADOS protocol. Eye contact is of special significance in two tasks, response to name call and joint attention, where it is used as a direct measure of a child's attention towards the robot. However, strict technical definition of the eye contact is lacking, especially in terms of duration and reciprocity. Authors in [11] expect that natural eye contact during conversation should last from 3 to 10 seconds. However, clinical practice and studies such as [12] suggest that children with ASD have significantly shorter attention span than typically developed children. In terms of reciprocity of eye contact, some authors [13] consider the eye contact to be established when both parties acknowledge the gaze direction of the other party, mainly through facial expressions. Considering the lack of controllable facial features on the NAO robots used in robot-assisted diagnostic protocol, we focus mainly on the recognition of eye contact in terms of gaze aimed towards NAO's eyes.

As reported in [14], looking at the eyes of others was significantly decreased in children (aged 2) with ASD, while looking at the mouth was increased compared to the control group of typically developed children. While other authors [15] suggest that the aforementioned difference was not statistically significant, this debate indicates that the technical system for eye contact detection needs to be precise and able to distinguish between child looking at the eyes of the robot and child looking at the other parts of the robot. Head mounted eye trackers can satisfy this requirement, but mounting them can be difficult with the children with ASD, especially due to the indispensable calibration procedure. 
Additionally, they require the object that the child is looking at to be stationary and are difficult to use on-line, rendering them unusable in the robot-assisted diagnostic protocol. To keep the protocol non-invasive, we are using the robot's camera for on-line detection of eye contact. However, methods for head pose and eye gaze estimation from a monocular camera in general do not possess the required precision to differentiate the child looking at different parts of the robot. Additionally, they usually produce a binary result for eye contact detection, which could be prone to false positives and negatives, which is detrimental for the reliable diagnosis of ASD.

To determine the quality of the observed eye contact and ensure continuous measure of attention throughout the diagnostic task, as a main contribution of the paper, we propose probabilistic eye contact detection, based on the sampling of Gaussian representations of head pose and gaze estimates.

The paper is organized as follows. Section 2 provides the overview of head pose and gaze estimation methods and their use for eye contact detection. In Section 3, we describe the probabilistic framework for eye contact detection, which takes into account both the probability and the duration of eye contact. Section 4 discusses results obtained in a laboratory setting. Conclusion and guidelines for future work are given in Section 5.

\section{HEAD POSE AND GAZE ESTIMATION FOR EYE CONTACT DETECTION}

The most straightforward way to approach eye contact detection is to use machine learning methods on a prelabeled set of images, avoiding the problem of 3D head pose and gaze estimation. Considering the eye contact detection within the robot-assisted ASD diagnostic protocol, the main drawback of such approach is not being able to take into account that the region of the eyes on the robot is not necessarily in the origin of the camera frame, reducing the accuracy of the training dataset. Additionally, if the approach is to be expanded towards additional regions of interest it would require the whole process of labeling and learning to be repeated.

According to [16], gaze combined with head pose and pointing gestures make significant contributions to the determination of another's focus of attention and subsequently the eye contact. Head pose and gaze estimation are active and prolific areas of research within the scientific community [17][18], and the results from those areas are often used to detect the focus of attention of individuals [19][20]. Herein, we use the output of those algorithms as inputs for the algorithm of eye contact detection we propose. To provide these inputs, we use commercially available face tracking and analysis software visage $\mid S D K$ [21]. Through a well documented API, visage $\mid S D K$ enables head pose, gaze, and facial-features tracking. It also provides $3 \mathrm{D}$ positions of all facial features, including the eyes which are the origins of the gaze. Gaze is estimated in terms of azimuth $\theta$ and elevation $\varphi$ with respect to the camera frame as a single measurement

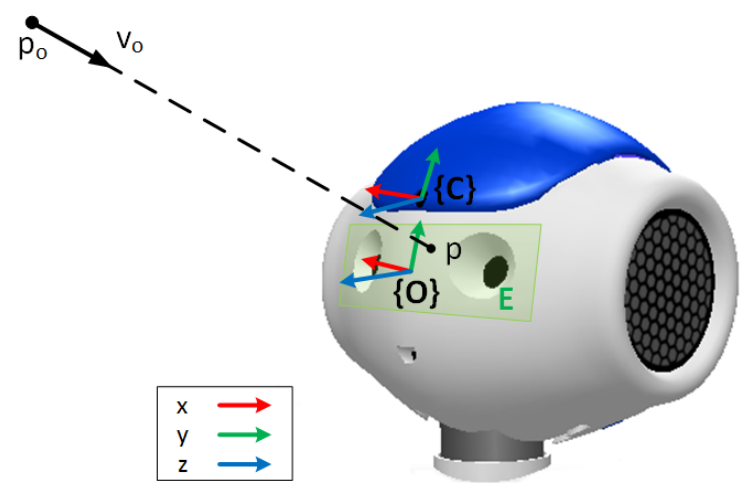

Fig. 1. Detecting eye contact with NAO robot using gaze origin $p_{o}$ and gaze direction $v_{o}$. Point $p$ is projection of $p_{o}$ along the vector $v_{o}$ on the plane $\pi_{o}$ spanned by $x$ and $y$ vectors of coordinate frame $\{O\}$. If point $p$ is inside eye region $\mathcal{E}$, eye contact is detected.

for both eyes. Therefore, we use the midpoint of the line connecting two eyes as the origin of gaze and denote it $\mathbf{p}_{c}$. For the upcoming calculations it is neccessary to transform the orientation of gaze into the unit vector of gaze direction $v_{c}=\left[r_{x}, r_{y}, r_{z}\right]$. Taking into account that the camera frame $\{C\}$ for the visage $\mid S D K$ is left-handed, following relations hold:

$$
\begin{aligned}
& r_{x}=-\sin (\theta) \\
& r_{y}=-\cos (\theta) \sin (\varphi) \\
& r_{z}=-\cos (\theta) \cos (\varphi)
\end{aligned}
$$

The origin of a subject's gaze $\mathbf{p}_{c}$ and unit gaze vector $\mathbf{v}_{c}$ are obtained from the tracking software in coordinate frame of the camera $\{C\}$. Let robot's eye region $\mathcal{E}$ lie in the plane $\pi_{o} \leftarrow A x+B y+C z+D=0$. Plane $\pi_{o}$ is defined in the coordinate frame of the eyes $\{O\}$, with known transformation between coordinate frames of the eyes and camera $\mathbf{T}_{o}^{c}$. Using $\mathbf{T}_{o}^{c}$, one can easily express $\mathbf{p}_{c}$ and $\mathbf{v}_{c}$ in the coordinates of $\{O\}$, obtaining origin of gaze $\mathbf{p}_{o}=\left[p_{x}, p_{y}, p_{z}\right]$ and gaze vector $\mathbf{v}_{o}=\left[v_{x}, v_{y}, v_{z}\right]$. Now, $\mathbf{p}_{o}$ and $\mathbf{v}_{o}$ define a line in $\{O\}$ (see Fig. 1), and to calculate the point $\mathbf{p}$ where this line intersects the plane $\pi_{o}$, we need to find $t \in \mathbb{R}$ such that the following holds:

$$
\mathbf{p}=\mathbf{p}_{o}+t \cdot \mathbf{v}_{o} \in \pi_{o}
$$

which can be rewritten as:

$$
A\left(p_{x}+t v_{x}\right)+B\left(p_{y}+t v_{y}\right)+C\left(p_{z}+t v_{z}\right)+D=0
$$

yielding the following solution for $t$ :

$$
t=-\frac{A p_{x}+B p_{y}+C p_{z}+D}{A v_{x}+B v_{y}+C v_{z}}
$$

Equation (4) has exactly one solution if the line defined by the gaze vector is not parallel with the plane $\pi_{o}$, which is the case in the intended application. Point $\mathbf{p}$ on the plane is calculated by plugging $t$ into (2), and eye contact is detected if that point is inside the region which corresponds to the eyes of the robot $\mathcal{E}$ (see Fig 1). 


\section{PROBABILISTIC EYE-CONTACT DETECTION}

To provide a continuous measure, we propose the conditional probability of eye contact, given the random vectors for head pose and gaze:

$$
p(X \mid H, G)
$$

$X$ is a random variable describing the eye contact, while $H$ and $G$ are normally distributed random vectors attached to head pose and gaze direction:

$$
\begin{aligned}
& H \sim \mathcal{N}\left(\mathbf{p} \in \mathbb{R}^{3}, \boldsymbol{\Sigma}_{p} \in \mathbb{R}^{3 x 3}\right) \\
& G \sim \mathcal{N}\left(\mathbf{o} \in \mathbb{R}^{2}, \boldsymbol{\Sigma}_{o} \in \mathbb{R}^{2 x 2}\right)
\end{aligned}
$$

with mean values $\mathbf{p}$ and $\mathbf{o}$ provided by the face tracking system. Variance matrices $\boldsymbol{\Sigma}$ of these random variables are a measure of the quality of the face tracking systems, i.e. they represent the confidence in the results of the estimation. Since visage|SDK employs extended Kalman filter to track facial features, the underlying variances matrices of the Kalman filter could be used directly, but are not available in the current version of the software. Therefore, we estimate the variance matrices during the calibration proces (see Section IV).

Knowing $H$ and $G$, our main goal is to obtain the probability density function of gaze direction on the plane $\pi_{o}$, similar to obtaining the point on the plane from Section II. Obtaining an analytical solution to this problem is the subject of our ongoing research. In this paper we provide a numerical solution based on sampling the distributions of $H$ and $G$.

First, we draw $N$ samples from $H$ and $M$ samples form $G$, generating $N \cdot M$ pairs of (point, orientation). For each of these pairs we calculate the gaze projection point on the plane $\pi_{o}$, as described in Section II. This procedure produces $N \cdot M$ samples in the plane $\pi_{o}$ (Fig. 2), which can be approximated by an arbitrary distribution through statistical analysis.

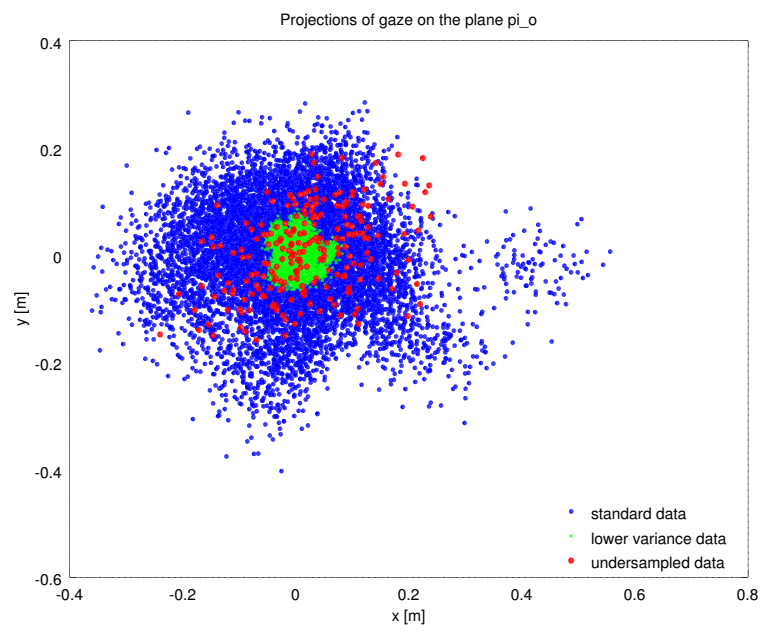

Fig. 2. Projection of the near vertical gaze on the plane $\pi_{o}\left(0^{\circ}\right.$, distance $1 \mathrm{~m})$
Since all of the samples are lying on a plane, we opt for bivariate normal distribution with the following probability density function:

$$
f(\mathbf{x}, \boldsymbol{\mu}, \boldsymbol{\Sigma})=\frac{1}{\sqrt{|\boldsymbol{\Sigma}|(2 \pi)^{2}}} e^{-\frac{(\mathbf{x}-\mu)^{\prime} \mathbf{\Sigma}^{-1}(\mathbf{x}-\boldsymbol{\mu})}{2}}
$$

where $\boldsymbol{\mu} \in \mathbb{R}^{2}$ is mean vector and $\boldsymbol{\Sigma} \in \mathbb{R}^{2 x^{2}}$ is covariance matrix.

As can be seen in Fig. 3, the set of points does not necessarily resemble Gaussian distribution for large angles of gaze, mainly due to the projective transformation which introduces nonlinearity in the mapping of distributions. However, as the field of view of the robot is relatively narrow and the head of the robot can be controlled to always face the subject during the interaction, gaze can never attain angles large enough for this effect to occur, which justifies use of normal distribution from (8).

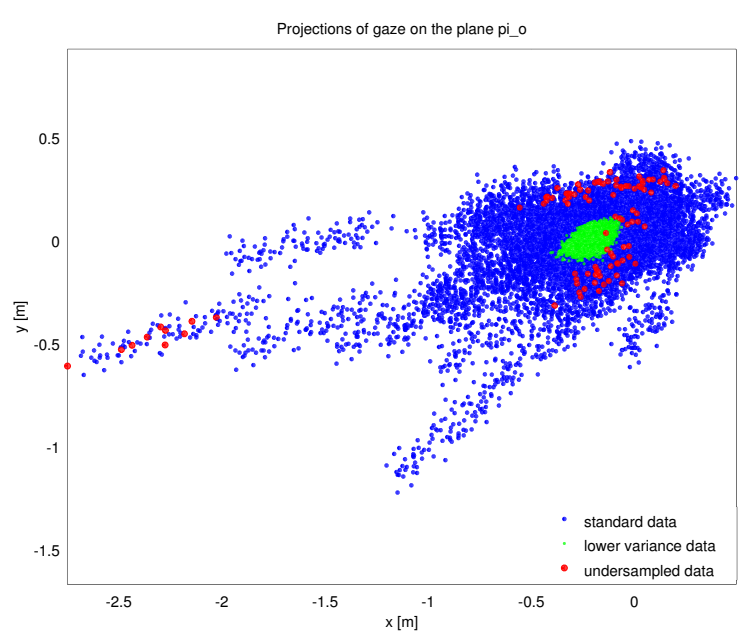

Fig. 3. Projection of the angled gaze on the plane $\pi_{o}\left(45^{\circ}\right.$, distance $\left.1.5 \mathrm{~m}\right)$

To obtain the probability of eye contact given the probability density function of gaze on the plane $\pi_{o}$, we need to integrate (8) over the region of the robot's eyes $\mathcal{E}$ :

$$
p(X=\text { eye contact })=\iint_{\mathcal{E}} f(\mathbf{x}, \boldsymbol{\mu}, \mathbf{\Sigma}) d \mathbf{x}
$$

Double integral in (9) cannot be analytically solved for an arbitrary area $\mathcal{E}$, therefore it needs to be evaluated numerically.

Along with the probabilty of the eye contact, it is also important to take into account the duration of the eye contact. As stated in the introductory section, studies usually compare durations between children with ASD and typically developed children, but do not state how long the eye contact needs to be before it is classified as eye contact, leaving the definition of eye contact incomplete, at least in technical terms. The main goal of introduction of the minimal duration is to eliminate the short glances which do not represent the complete transfer of attention towards robot's eyes. According to observations in clinical practice which state 
that the eye contact duration for children with ASD is short in comparison with typically developed children, we set this duration to $0.5 \mathrm{~s}$. To incorporate this temporal dimension of the eye contact into the proposed probabilistic framework, we introduce continuous first order filter of the following form:

$$
G(s)=\frac{1}{T \cdot s+1}
$$

where $T$ is the time constant of the filter. We set $T$ such that the filter from (10) reaches $95 \%$ of the final value within $0.5 \mathrm{~s}$, which equates to $T=0.15 \mathrm{~s}$. Next, we discretize the filter using bilinear transform, taking into account sample time $T_{s}$ which is determined by the framerate of the camera and may vary depending on the resolution of the video stream:

$$
G(z)=\frac{T_{s} \cdot z+T_{s}}{\left(2 T+T_{s}\right) \cdot z+\left(T_{s}-2 T\right)}
$$

From (11) stems the update equation of the first order discrete filter:

$$
y_{k}=\frac{1}{2 T+T_{s}}\left[\left(2 T-T_{s}\right) y_{k-1}+T_{s}\left(x_{k}+x_{k-1}\right)\right]
$$

where subscript $k$ denotes values in the current time step, while $k-1$ denotes the values from the previous time step.

\section{RESULTS}

The experiments described in this section are performed by recording the video using the camera of NAO robot with resolution $640 \times 480$ pixels. Video is then processed on the computer running visage $\mid S D K$. While visage $\mid S D K$ has proven to be accurate in terms of tracking, during the experiments we observed an error in the initialization step of the tracker which resulted in an offset in the estimate of the elevation angle $\varphi$. This offset is introduced when fitting 3D model to facial features and is observed to be constant for one recording, but may vary between different recordings. Therefore, we introduce the calibration step in each of the experiments. Calibration consists of looking directly at the camera of the robot for the predefined amount of time in the start of the experiment, which enables the tracker to calculate the offset knowing that the subject is looking directly at the camera. During this calibration step we also calculate and record the variances in the head pose and gaze data. Averaging these variances over multiple calibration steps yields the following matrices which are used in the experiments:

$$
\begin{aligned}
& \boldsymbol{\Sigma}_{p}=\left[\begin{array}{ccc}
0.010 & 0 & 0 \\
0 & 0.0005 & 0 \\
0 & 0 & 0.006
\end{array}\right] \\
& \boldsymbol{\Sigma}_{o}=\left[\begin{array}{cc}
0.017533 & 0 \\
0 & 0.015020
\end{array}\right]
\end{aligned}
$$

In the experiments we use $N=100$ and $M=100$, obtaining 10000 samples from which the projected distribution is computed. Finally, the integral from (9) is numerically computed over eye region (green rectangle in Fig. 4) using GaussLegendre quadrature. Obtained probability is then filtered through (11), with sample time $T_{s}=0.077 \mathrm{~s}$, corresponding to 13 FPS which is the maximum for the NAO camera at the resolution of $640 \times 480$ pixels. With the aforementioned parameters, the average runtime of the algorithm for the probability calculation is under 2 milliseconds (not taking into account the time needed for visage $\mid S D K$ to estimate head pose and gaze).

\section{A. Transfer of attention}

This experiment is designed to evaluate the ability of the proposed probabilistic framework to detect the transfer of attention of the subject towards the eyes of the robot. We instruct the subject to focus on seven different areas (Fig 4) of the robot, starting with the camera region $\mathcal{C}$ to obtain the calibration parameters. Next the subject needs to focus on the eyes region $\mathcal{E}$, denoted with green rectangle in Fig. 4, size of which is $10 \times 4 \mathrm{~cm}$.

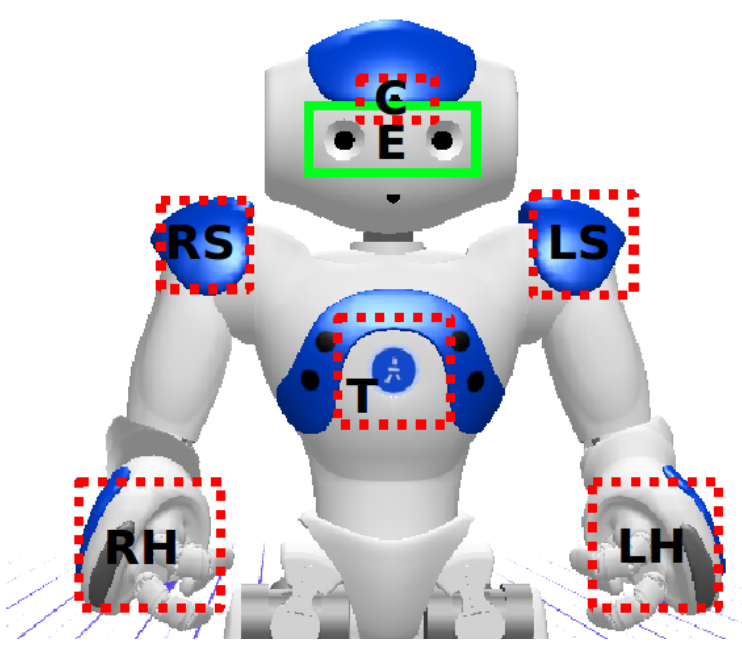

Fig. 4. Regions of interest during the attention transfer experiment.

After focusing on the eyes of the robot, the subject needs to transfer attention to the torso $\mathcal{T}$, left hand $\mathcal{L} \mathcal{H}$, right hand $\mathcal{R H}$, right shoulder $\mathcal{R S}$ and left shoulder $\mathcal{L S}$ in a sequence. After the left shoulder, the subject is instructed to look towards robot's eyes once again. The results are shown in Fig. 5.

The plot of the probability of eye contact in Fig. 5 suggests that the proposed framework has the capability to detect eye contact with high probability and is not prone to false positives when looking at the other parts of the robot, with the exception of camera region which is expected due to the partial overlap of the regions.

Next, we lower confidence in the head pose and gaze estimation by doubling the values of variance matrices from (13). The results are shown in Fig. 6.

By comparing the probabilities from Fig. 5 and Fig. 6, one can observe that lower confidence in head pose and gaze estimates results in decrease of the probability of eye contact, meaning that the robot has less confidence that the eye contact really occurred. 


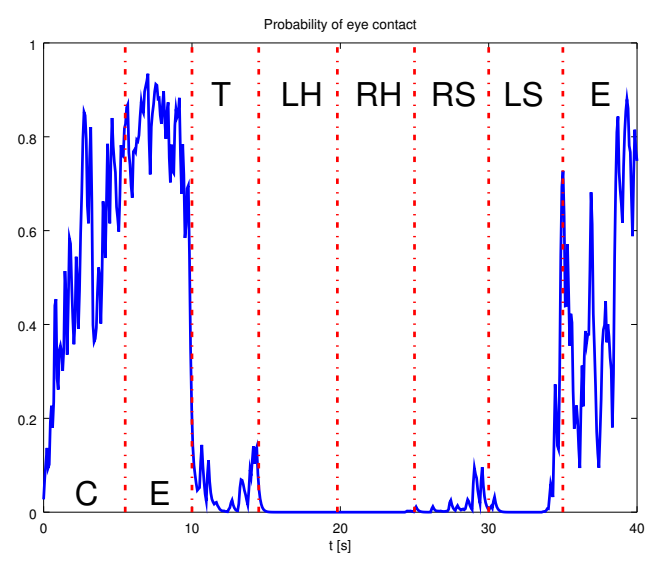

Fig. 5. Probability of eye contact during the experiment. Subject is $56 \mathrm{~cm}$ from the robot and looking at camera $\mathcal{C} \rightarrow$ eyes $\mathcal{E} \rightarrow$ torso $\mathcal{T} \rightarrow$ left hand $\mathcal{L} \mathcal{H} \rightarrow$ right hand $\mathcal{R H} \rightarrow$ right shoulder $\mathcal{R S} \rightarrow$ left shoulder $\mathcal{L} \mathcal{S} \rightarrow$ eyes $\mathcal{E}$.

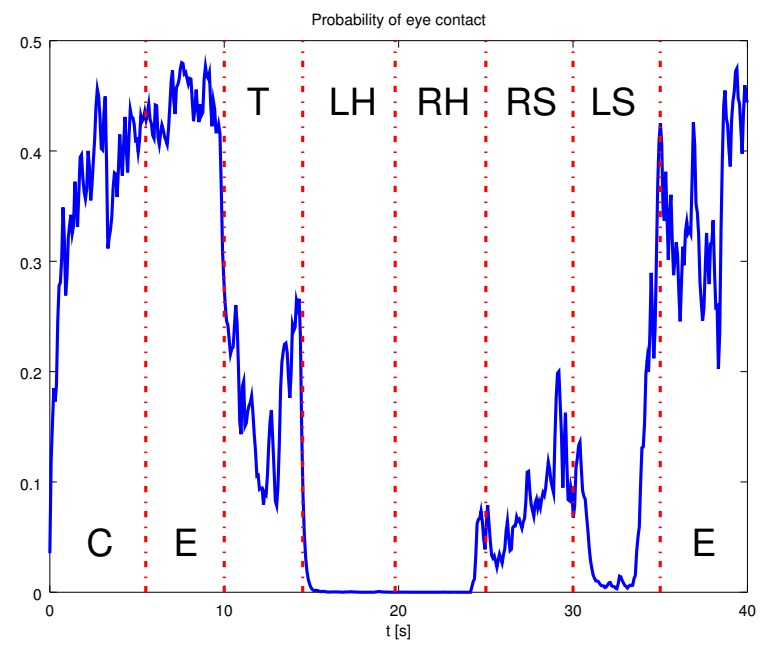

Fig. 6. Probabilty of eye contact with lower confidence in head pose and gaze estimation. Subject is $56 \mathrm{~cm}$ from the robot and looking at camera $\mathcal{C} \rightarrow$ eyes $\mathcal{E} \rightarrow$ torso $\mathcal{T} \rightarrow$ left hand $\mathcal{L} \mathcal{H} \rightarrow$ right hand $\mathcal{R H} \rightarrow$ right shoulder $\mathcal{R S} \rightarrow$ left shoulder $\mathcal{L} \mathcal{S} \rightarrow$ eyes $\mathcal{E}$.

\section{B. Evaluating glances}

This experiment is designed to assess the proposed frame work with respect to short glances towards the robots eyes, which should not be classified as eye contact. After the calibration step, the subject is instructed to avert gaze from the robot and to produce several short glances towards the robot eyes without focusing on them. The computed probability for this experiment is shown in Fig. 7 .

Fig. 7 shows the effect of the discrete filter on the computed probability. Without the filter, first short glance would be classified as eye contact with $90 \%$ confidence, while with filter the probability reaches only $35 \%$. The effect is that the robot has less confidence that the eye contact has occurred for short glances, diminishing the possibility of falsely classifying glances as eye contact.

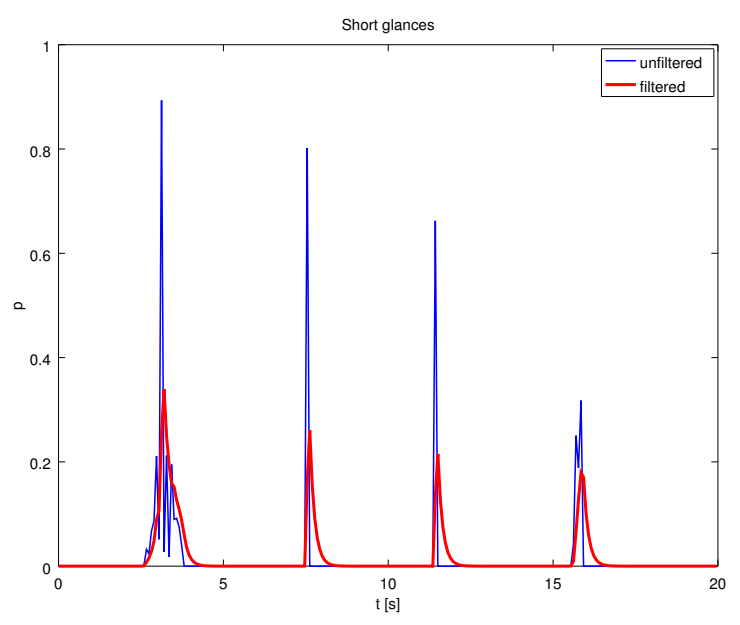

Fig. 7. Probability of eye contact for short glances towards robot's eyes.

\section{Distance from the robot}

This experiment is designed to assess the calculated eye contact probability with respect to the distance of the subject from the robot. The subject was instructed to maintain eye contact while robot was approaching the subject and then moving away, and the calculated probability is shown in Fig. 8.
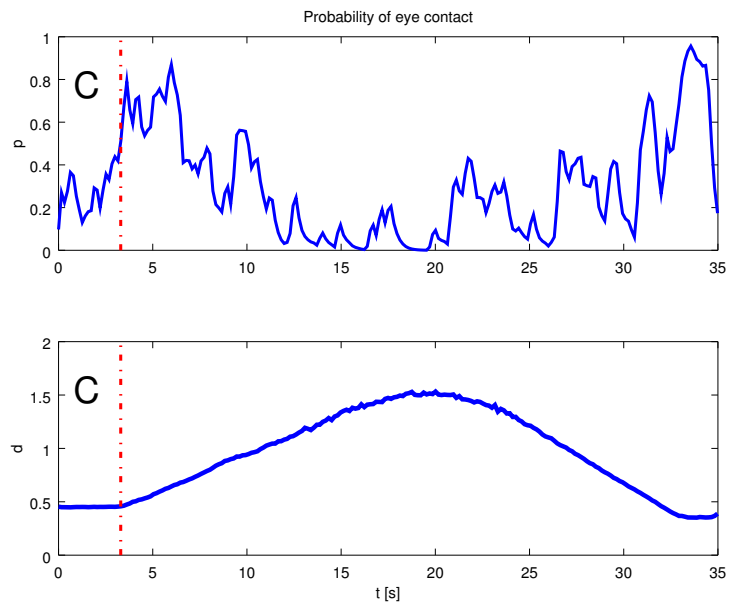

Fig. 8. Probability of eye contact with respect to distance. For first $3.5 \mathrm{~s}$ subject is looking at the camera $\mathcal{C}$ to calibrate the tracking system.

After the initial calibration period, the trend of probability of eye contact in Fig. 8 is inversely proportional to the distance from the robot. With larger distance, small variations in head pose and gaze result in larger offsets on the plane $\pi_{o}$, spreading the distribution, thus decreasing the probability over the fixed region. Similar effect is observed when varying the angle of the gaze.

D. Undersampling the distributions of head pose and gaze

To illustrate the effect of $M$ and $N$ being too small to correctly represent the projected distribution, we analyze the 
recording of experiment from section IV-A with different values for $M$ and $N$. We run the analysis 3 times, showing the calculated probability in Fig. 9.

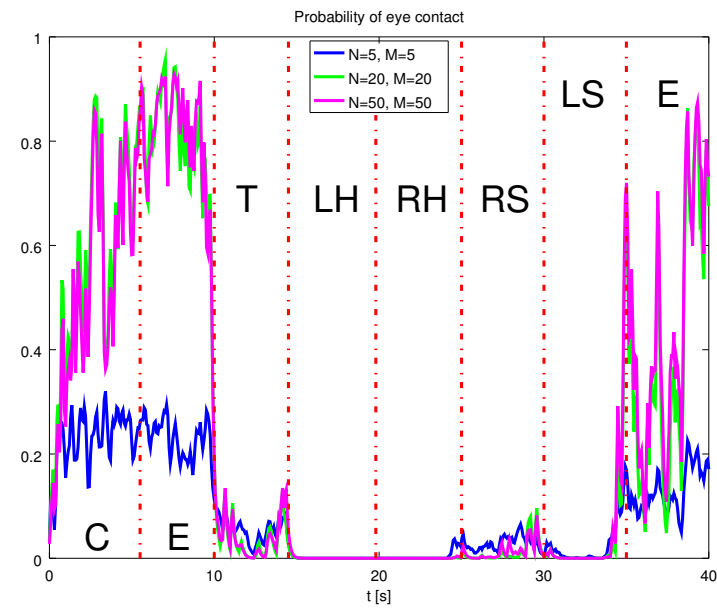

Fig. 9. Probability of eye contact for 3 runs of the algorithm with differen number of samples drawn from distributions of head pose and gaze. Subject is $56 \mathrm{~cm}$ from the robot and looking at camera $\mathcal{C} \rightarrow$ eyes $\mathcal{E} \rightarrow$ torso $\mathcal{T} \rightarrow$ left hand $\mathcal{L} \mathcal{H} \rightarrow$ right hand $\mathcal{R H} \rightarrow$ right shoulder $\mathcal{R S} \rightarrow$ left shoulder $\mathcal{L S} \rightarrow$ eyes $\mathcal{E}$.

Comparing Fig. 5 and Fig. 9, it can be observed that small number of samples cannot represent the projected distribution adequately, resulting in the inconsistent calculation of the probability of eye contact. Although there are no significant differences between plots for number of samples larger than 20 for the given experiment, minimal number of samples increases with increased variance in estimates of head pose and gaze.

\section{CONCLUSION}

To reliably detect eye contact, a key feature in envisioned robot-assisted ASD diagnostic protocol, we propose a general probabilistic framework based on sampling of normal distributions of head pose and gaze estimates. The proposed framework provides a continuous measure of eye contact in form of probability, with increased robustness to outliers. It also takes into account temporal component of the eye contact, diminishing the possibility of false detections of eye contact by discarding short glances. Through several experiments in a laboratory setting, we show that the proposed framework can successfully cope with variances in the estimates of head pose and gaze.

Analytical solution to the distribution projection problem is of highest importance in our future work, as is the assessment of the proposed probabilistic framework in clinical sessions with children. We plan to incorporate the proposed method into the robot-assisted diagnostic protocol to deduce whether the eye scanning patterns during the interaction with NAO can be used to more acurately differentiate children with ASD and typically developed children.

\section{ACKNOWLEDGMENT}

This work has been fully supported by Croatian Science Foundation under the project Autism Diagnostic Observation with Robot Evaluator (no. 93743-2014).

\section{REFERENCES}

[1] Diagnostic and Statistical Manual of Mental Disorders. American Psychiatric Association, 4th edition, 2002.

[2] C. Lord, M. Rutter, P.C. Dilavore, and S. Risi. Autism Diagnostic Observation Schedule. Western Psychological Services, 2002.

[3] M. Rutter, A. LeCouteur, and C. Lord. The Autism Diagnostic interview, Revised (ADI-R). Western Psychological Services, 2003.

[4] A. Klin, J. Lang, V. Chicchetti, and F.R. Volkmar. Interrater reliability of clinical diagnosis and DSM-IV criteria for autistic disorder: results of the DSM-IV autism field trial. Journal of Autism and Developmental Disorders, 30(2):163-167, 2000.

[5] F. R. Volkmar, C. Lord, A. Bailey, R. T. Schultz, and A. Klin. Autism and pervasive developmental disorders. Journal of Child Psychology and Psychiatry, 45:135170, 2004.

[6] I. Iacono, H. Lehmann, P. Marti, B. Robins, and K. Dautenhahn. Robots as social mediators for children with Autism - A preliminary analysis comparing two different robotic platforms. In Development and Learning (ICDL), 2011 IEEE International Conference on, volume 2, pages 1-6, 2011.

[7] Changchun Liu, K. Conn, N. Sarkar, and W. Stone. Online affect detection and robot behavior adaptation for intervention of children with autism. Robotics, IEEE Transactions on, 24(4):883-896, 2008.

[8] D. Maurer. Social Perception in Infants, chapter Infants perception of facedness. 1985.

[9] F. Petric, K. Hrvatinic, A. Babic, L. Malovan, D. Miklic, Z. Kovacic, M. Cepanec, J. Stosic, and S. Simlesa. Four tasks of a robot-assisted autism spectrum disorder diagnostic protocol: First clinical tests. In Global Humanitarian Technology Conference (GHTC), 2014 IEEE, pages 510-517, Oct 2014

[10] F. Petric, D. Tolić, D. Miklić, Z. Kovačić, M. Cepanec, and S. Šimleša. Intelligent Robotics and Applications: 8th International Conference, ICIRA 2015, Portsmouth, UK, August 24-27, 2015, Proceedings, Part II, chapter Towards A Robot-Assisted Autism Diagnostic Protocol: Modelling and Assessment with POMDP, pages 82-94. Springer International Publishing, Cham, 2015.

[11] Janet Dean Michael Argyle. Eye-contact, distance and affiliation. Sociometry, 28(3):289-304, 1965.

[12] G. Dawson, K. Toth, R. Abbott, J. Osterling, J. Munson, A. Estes, and J. Liaw. Early social attention impairments in autism: social orienting, joint attention, and attention to distress. Dev Psychol, 40(2):271-283, Mar 2004.

[13] C. L. Kleinke. Gaze and eye contact: a research review. Psychol Bull, 100(1):78-100, Jul 1986.

[14] W. Jones, K. Carr, and A. Klin. Absence of preferential looking to the eyes of approaching adults predicts level of social disability in 2-yearold toddlers with autism spectrum disorder. Arch. Gen. Psychiatry, 65(8):946-954, Aug 2008.

[15] T. Falck-Ytter and C. von Hofsten. How special is social looking in ASD: a review. Prog. Brain Res., 189:209-222, 2011.

[16] S. R. Langton, R. J. Watt, and I. Bruce. Do the eyes have it? Cues to the direction of social attention. Trends Cogn. Sci. (Regul. Ed.), 4(2):50-59, Feb 2000

[17] E. Murphy-Chutorian and M. M. Trivedi. Head pose estimation in computer vision: A survey. IEEE Transactions on Pattern Analysis and Machine Intelligence, 31(4):607-626, April 2009.

[18] D. W. Hansen and Q. Ji. In the eye of the beholder: A survey of models for eyes and gaze. IEEE Transactions on Pattern Analysis and Machine Intelligence, 32(3):478-500, March 2010.

[19] F. Vicente, Z. Huang, X. Xiong, F. De la Torre, W. Zhang, and D. Levi. Driver gaze tracking and eyes off the road detection system. IEEE Transactions on Intelligent Transportation Systems, 16(4):2014-2027, Aug 2015.

[20] R. Valenti, N. Sebe, and T. Gevers. Combining head pose and eye location information for gaze estimation. IEEE Transactions on Image Processing, 21(2):802-815, Feb 2012.

[21] Visage Technologies. visage|SDK. Available at http: //visagetechnologies.com/products-and-services/ visagesdk/, version used 7.4.25, accessed March, 2016. 